\title{
Antibacterial Activity of a Novel Peptide-Modified Lysin Against Acinetobacter baumannii and Pseudomonas aeruginosa
}

\author{
Hang Yang ${ }^{\dagger}$, Mengyue Wang ${ }^{\dagger}$, Junping Yu and Hongping Wei* \\ Key Laboratory of Special Pathogens and Biosafety, Center for Emerging Infectious Diseases, Wuhan Institute of Virology, \\ Chinese Academy of Sciences, Wuhan, China
}

The global emergence of multidrug-resistant (MDR) bacteria is a growing threat to

OPEN ACCESS

Edited by:

Rustam Aminov,

Technical University of Denmark,

Denmark

Reviewed by:

Manuela Caniça

National Institute of Health Doutor

Ricardo Jorge, Portugal

Maia Merabishvili,

Queen Astrid Military Hospital,

Belgium

Yves Briers,

Ghent University, Belgium

*Correspondence:

Hongping Wei

hpwei@wh.iov.cn

tThese authors have contributed equally to this work.

Specialty section:

This article was submitted to Antimicrobials, Resistance

and Chemotherapy,

a section of the journal

Frontiers in Microbiology

Received: 10 September 2015 Accepted: 07 December 2015

Published: 22 December 2015

Citation:

Yang $H$, Wang $M, Y u J$ and Wei $H$ (2015) Antibacterial Activity of a Novel Peptide-Modified Lysin Against Acinetobacter baumannii and

Pseudomonas aeruginosa.

Front. Microbiol. 6:1471. doi: 10.3389/fmicb.2015.01471 public health worldwide. Natural bacteriophage lysins are promising alternatives in the treatment of infections caused by Gram-positive pathogens, but not Gram-negative ones, like Acinetobacter baumannii and Pseudomonas aeruginosa, due to the barriers posed by their outer membranes. Recently, modifying a natural lysin with an antimicrobial peptide was found able to break the barriers, and to kill Gram-negative pathogens. Herein, a new peptide-modified lysin (PlyA) was constructed by fusing the cecropin A peptide residues 1-8 (KWKLFKKI) with the OBPgp279 lysin and its antibacterial activity was studied. PlyA showed good and broad antibacterial activities against logarithmic phase $A$. baumannii and $P$. aeruginosa, but much reduced activities against the cells in stationary phase. Addition of outer membrane permeabilizers (EDTA and citric acid) could enhance the antibacterial activity of PlyA against stationary phase cells. Finally, no antibacterial activity of PlyA could be observed in some bio-matrices, such as culture media, milk, and sera. In conclusion, we reported here a novel peptide-modified lysin with significant antibacterial activity against both logarithmic (without OMPs) and stationary phase (with OMPs) $A$. baumannii and $P$. aeruginosa cells in buffer, but further optimization is needed to achieve broad activity in diverse bio-matrices.

Keywords: bacteriophage lysin, engineered lysin, Acinetobacter baumannii, Pseudomonas aeruginosa, outer membrane permeabilizers (OMPs)

\section{INTRODUCTION}

The global emergence of multidrug-resistant (MDR) Gram-negative bacteria is a growing threat to public health worldwide ( $\mathrm{Li}$ et al., 2015). Due to the diversity of resistance mechanisms that may lead to MDR or even pandrug resistance (PDR; Livermore and Woodford, 2006; Potron et al., 2015), Acinetobacter baumannii and Pseudomonas aeruginosa are among the increasingly reported and commonly identified MDR or even PDR nosocomial pathogens. They are responsible for many hospital-acquired infections, ranging from mild skin wounds and urinary tract infections to severe life-threatening infections, including bloodstream, pneumonia, and meningitis (GarciaQuintanilla et al., 2013; Bassetti et al., 2014). At the same time, the progress in developing new antibiotics against these pathogens is slow (Fischbach and Walsh, 2009). Therefore, new strategies for controlling these MDR pathogens are urgently needed. 
Bacteriophage lysins, the weapon of phages to digest the host bacterial cell wall for the release of progeny phages, have been extensively demonstrated to be promising alternatives in the treatment of Gram-positive pathogens, such as staphylococci and streptococci (Nelson et al., 2012; Pastagia et al., 2013; Yang et al., 2014). Due to their unique working mechanisms, lysins possess a low possibility of developing resistance (Fischetti, 2008; Knoll and Mylonakis, 2014). However, the outer membranes of Gram-negative bacteria block the access of natural lysins to their peptidoglycan substrates, thus making the exogenously added lysins useless or very weak against the viability of the target cells (Morita et al., 2001; Lai et al., 2011; Lim et al., 2014).

Currently, several strategies have been developed to break the barriers posed by the outer membranes of Gram-negative bacteria to natural lysins. Physical (i.e., high hydrostatic pressure; Briers et al., 2008) and chemical permeabilizers (i.e., EDTA, and weak organic acid, usually citric acid; Briers et al., 2007, 2011) can permeabilize the outer membrane efficiently to enhance the antibacterial activity of lysins, but are only applicable in applications such as food conservation and the treatment of topical infections. Structure-based engineering and phage genome-based screening methods have also been used to find novel lysins that act on Yersinia with the FyuA receptor (Lukacik et al., 2012) and A. baumannii (Lood et al., 2015). In recent years, a few engineered lysins have been reported with good antibacterial activities against $P$. aeruginosa by fusing natural lysins with optimized $\mathrm{N}$ - or C-terminal lipopolysaccharidesdestabilizing or antimicrobial peptides, respectively, which can permeabilize the outer membranes (Briers et al., 2014a,b). In principle, considering the easy availability of lipopolysaccharidedestabilizing and antimicrobial peptides, fusing natural lysins with such peptides looks quite attractive since it provides a good way with plenty of chances to create novel engineered lysins against Gram-negative bacteria. However, reports on peptidemodified lysins are limited, and mainly focus on P. aeruginosa.

In the present study, a new peptide-modified lysin (PlyA) against $A$. baumannii and $P$. aeruginosa was constructed by fusing the cecropin A peptide residues 1-8 (KWKLFKKI) with the OBPgp279 lysin (Walmagh et al., 2012), and its antibacterial activity was evaluated.

\section{MATERIALS AND METHODS}

\section{Bacterial Strains}

All bacterial strains and clinical isolates (Table 1) used in this work were grown in Luria Broth (LB) medium at $37^{\circ} \mathrm{C}$. All clinical isolates of $A$. baumannii and $P$. aeruginosa were identified by $16 \mathrm{~S}$ rDNA sequencing analysis combined with the biochemistry test using a MicroStation system (Biolog, GEN III Omnilog Combo Plus System, USA).

\section{Construction of Plasmids}

Cecropin $\mathrm{A}$ is a 37 -residue membrane-active antimicrobial polypeptide that kills bacteria by dissipating transmembrane electrochemical ion-gradients (Silvestro and Axelsen, 2000). Because the N-terminal residues $1-8$ of cecropin A (CA,
KWKLFKKI) are highly positively charged, this fragment was used in this study. OBPgp279 coding sequence was initially cloned into the modified pET28a $(+)$ plasmid $\left(\operatorname{Kan}^{\mathrm{R}}\right)$ containing a $(\mathrm{G} 4 \mathrm{~S})_{2}$ liner between BamHI and EcoRI sites (Yang et al., 2012), using primers OBP-F (5-tatagaattcatgaaaaactcggaaaagaacg-3) and OBP-R (5-atatctcgagcacgatacccagagcttttttg-3), to obtain pET-OBPgp279 vector $\left(\operatorname{Kan}^{\mathrm{R}}\right)$. The coding sequence for cecropin A peptide residues 1-8 (CA, KWKLFKKI) was cloned into the pET-OBPgp279 vector, using primers CA-F (5-catgggcaaatggaaattatttaagaaaattg-3) and CA-R (5gatccaattttcttaaataatttccatttgcc-3), to obtain pET-CA-OBPgp279 (pET-PlyA, $\operatorname{Kan}^{\mathrm{R}}$ ) vector. After verification by sequencing, E. coli BL21(DE3) cells were transformed with the correct plasmid for protein expression.

\section{Protein Purification}

The recombinant enzymes were purified as described previously in our laboratory (Huang et al., 2015). Briefly, the E. coli BL21(DE3) cells were induced with $0.5 \mathrm{mM}$ isopropyl $\beta$-Dthiogalactoside (IPTG) overnight at $16^{\circ} \mathrm{C}$ and collected for protein purification after sonication on ice. Then the proteins were collected by washing and eluting with 80 and $400 \mathrm{mM}$ imidazole through a nickel nitrilotriacetic acid column (GE Healthcare, US), respectively. The collected active protein fractions were pooled and dialyzed against $20 \mathrm{mM}$ Tris- $\mathrm{HCl}(\mathrm{pH}$ 7.4) and stored at $-80^{\circ} \mathrm{C}$ until use (less than 2 weeks).

\section{Antibacterial Activity Assay}

To determine the antibacterial activity of PlyA, logarithmic (cultured for 3-4 h, $\mathrm{OD}_{600}=0.6-0.8$ ) or stationary phase (cultured for $14-16 \mathrm{~h}, \mathrm{OD}_{600}=1.4-1.6$ ) cultures of $A$. baumannii WHG3066 were centrifuged $(10,000 g \times 1 \mathrm{~min})$ first. Then the cells were washed once and resuspended in $20 \mathrm{mM}$ Tris- $\mathrm{HCl}(\mathrm{pH}$ 7.4). Bacterial suspensions $(100 \mu \mathrm{l})$ were mixed with the enzyme in the presence or absence of the outer membrane permeabilizers (OMPs, i.e., EDTA and citric acid) at $37^{\circ} \mathrm{C}$ for $15-60 \mathrm{~min}$. Finally, the remaining viable cells were calculated by plating onto LA plates. For susceptibility test, clinical isolates of A. baumannii and $P$. aeruginosa were cultured to logarithmic phase and treated with 50 (for A. baumannii isolates) or $100 \mu \mathrm{g} / \mathrm{ml}$ (for P. aeruginosa isolates) $\mathrm{PlyA}$ at $37^{\circ} \mathrm{C}$ for $1 \mathrm{~h}$. All assays were performed for at least three times in biological repeats.

To test the synergism between OMPs and PlyA, stationary phase A. baumannii WHG3066 and P. aeruginosa WHG3012 cells were treated with $100 \mu \mathrm{g} / \mathrm{ml}$ PlyA in the presence of various concentrations of EDTA or citric acid in $20 \mathrm{mM}$ Tris- $\mathrm{HCl}(\mathrm{pH}$ 7.4) or $5 \mathrm{mM}$ HEPES-NaOH ( $\mathrm{pH} 7.4$ ) at $37^{\circ} \mathrm{C}$ for $1 \mathrm{~h}$. Afterward, the viable cell numbers were counted by plating. To avoid the acidification effect of citric acid, the synergism between citric acid and PlyA was also performed by adjusting the $\mathrm{pH}$ values of the reaction systems to 7.4. All assays were performed for at least three times in biological repeats.

To test the effects of bio-matrix on the antibacterial activity of PlyA, A. baumannii WHG3066 cells in logarithmic phase were washed once with $20 \mathrm{mM}$ Tris- $\mathrm{HCl}$ buffer ( $\mathrm{pH} 7.4$ ), and resuspended in medium, including LB, Mueller-Hinton $(\mathrm{MH}$, Huankai Microbial, Guangdong, China), Brain Heart Infusion 
TABLE 1 | Bacterial strains and clinical isolates used in this work.

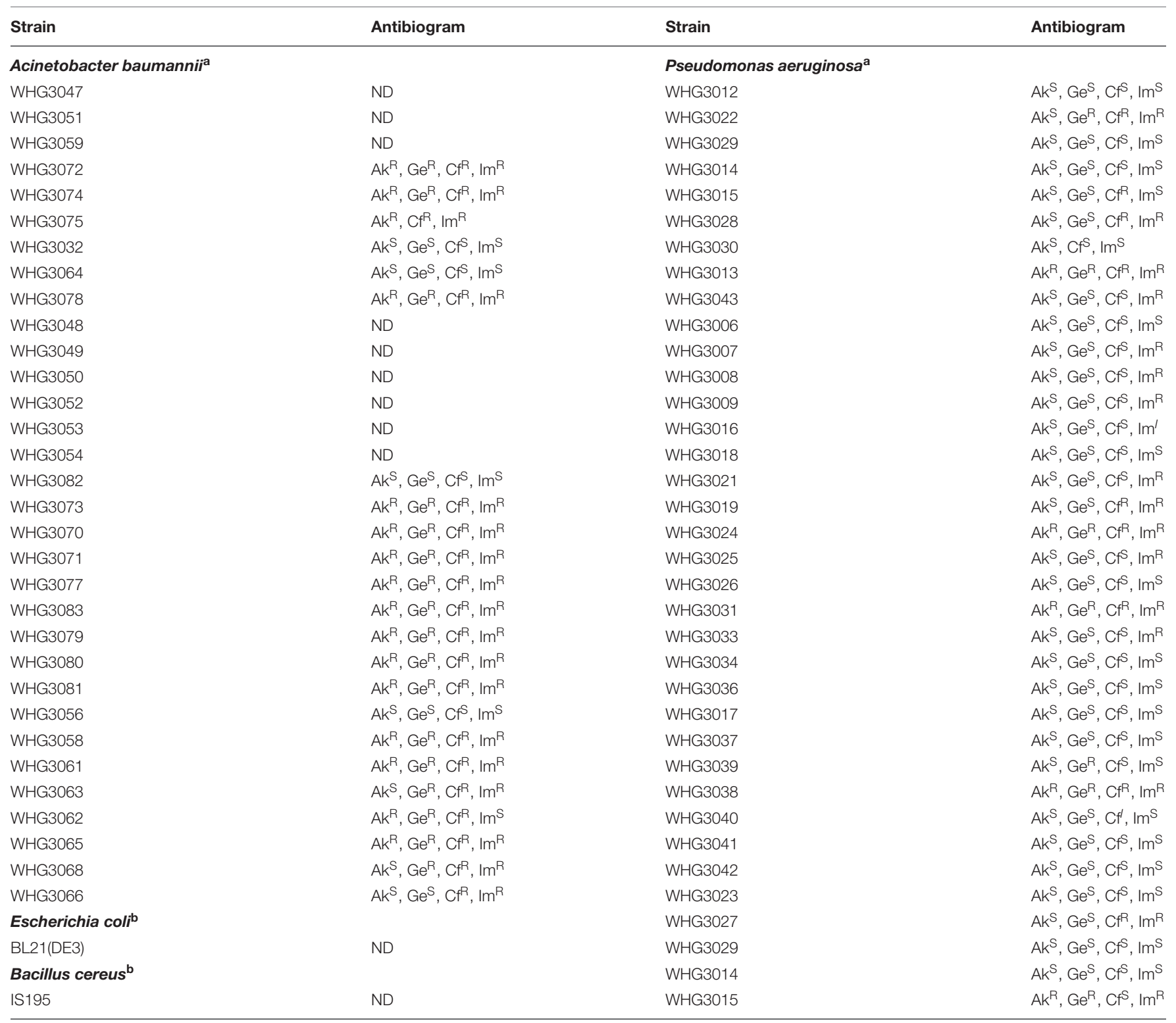

Antibiogram: Ak, amikacin; Ge, gentamicin; Cf, cefepime; Im, imipenem; ND, not detected; R, resistant; S, susceptible; I, intermediate-resistant.

a/solated from Zhongnan Hospital of Wuhan University; ${ }^{b}$ Lab collection.

(BHI, Huankai Microbial, Guangdong, China) and Tryptic Soy Broth (TSB, Becton, Dickinson \& Co., France) with $4 \% \mathrm{NaCl}$ (TSBN), or pasteurized milk (Mengniu Group, Wuhan, China), or human serum (Sigma-Aldrich, Shanghai, China). Then, cells were treated with $100 \mu \mathrm{g} / \mathrm{ml} \mathrm{PlyA}$ at $37^{\circ} \mathrm{C}$ for $1 \mathrm{~h}$, respectively. The viable cell numbers were evaluated by plating on LA plates. All assays were performed for at least three times in biological repeats.

\section{Transmission Electron Microscope (TEM)}

The action of PlyA on the cell wall of the bacteria was monitored by a thin-section transmission electron microscope (Tecnai $\mathrm{G}^{2}$ 20 TWIN, FEI, USA). Briefly, A. baumannii WHG3066 cell suspensions in logarithmic phase were incubated with $100 \mu \mathrm{g} / \mathrm{ml}$
PlyA at $37^{\circ} \mathrm{C}$ for 10,15 , and $30 \mathrm{~min}$, respectively. Then, the reactions were terminated by addition of $2.5 \%$ glutaraldehyde and the fixed samples were analyzed by transmission electron microscope (TEM). Cells treated with 20 mM Tris- $\mathrm{HCl}$ ( $\mathrm{pH} 7.4$ ) under the same conditions were used as controls.

\section{RESULTS}

\section{Characteristics of PlyA in Tris-HCI Buffer}

As shown in Figure 1A, CA-fused OBPgp279 (called PlyA for short) and its parental lysin OBPgp279 were well expressed in E. coli as soluble proteins with purity of $>95 \%$ as observed by $12 \%$ SDS-PAGE gel. Antibacterial activity tests showed that 

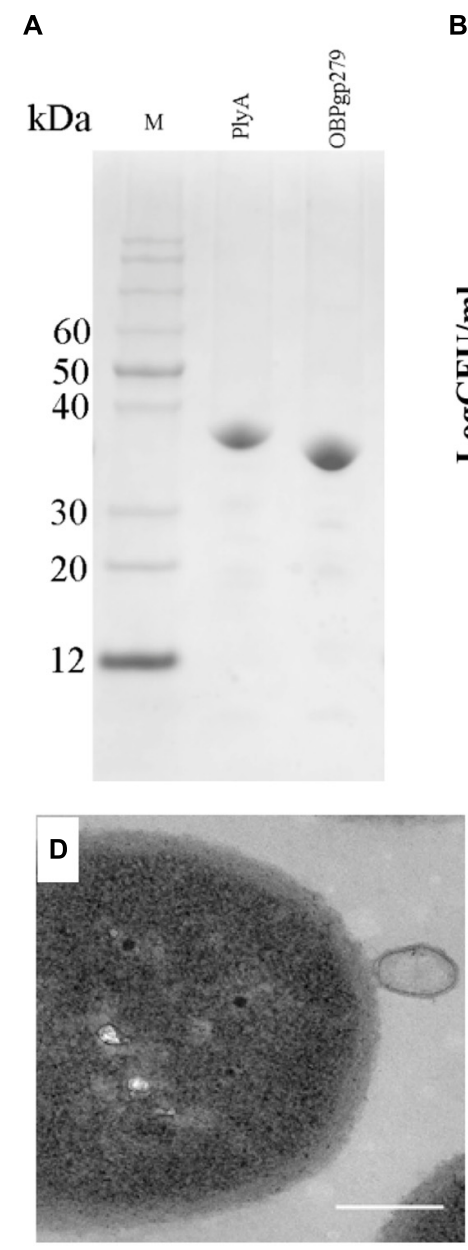

B

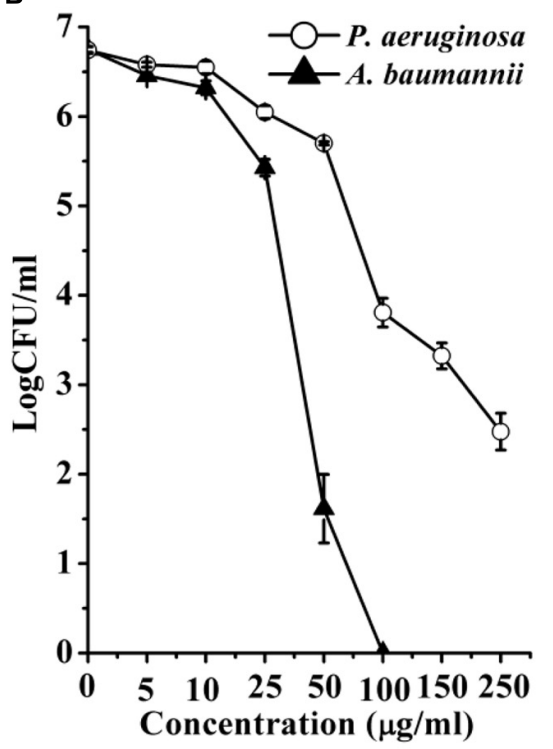

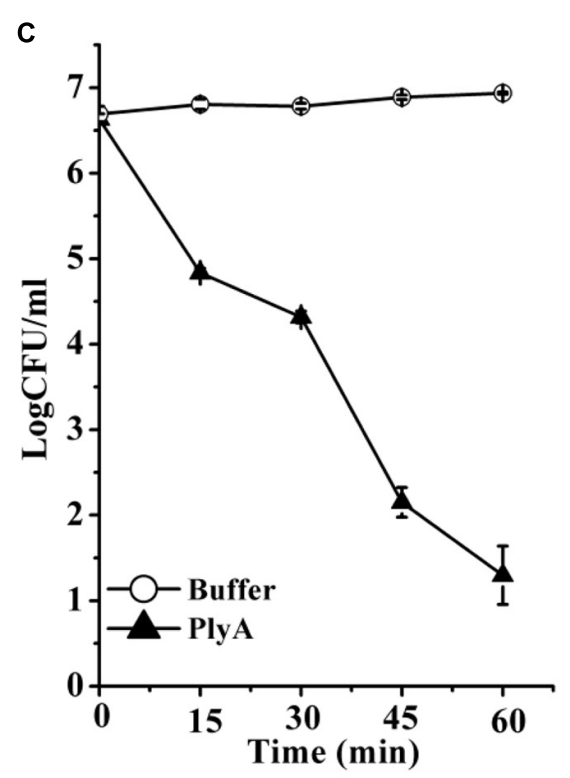
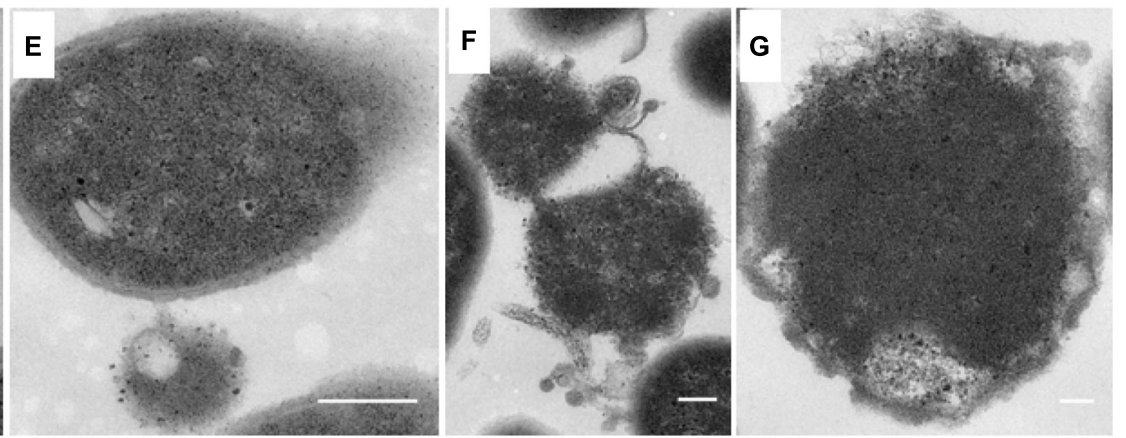

FIGURE 1 | Activity of peptide-modified lysin (PlyA) in Tris-HCI buffer. (A) Analysis of purified proteins on 12\% SDS-PAGE gel. (B) Dose-dependent antibacterial activity of PlyA against logarithmic Acinetobacter baumannii WHG3066 and Pseudomonas aeruginosa WHG3012 in 20 mM Tris- HCl (pH 7.4). (C) Time-killing curve of $50 \mu \mathrm{g} / \mathrm{ml}$ PlyA against logarithmic A. baumannii WHG3066. (D-G) Transmission electron microscope (TEM) images of logarithmic A. baumannii WHG3066 exposed to PlyA. TEM analysis revealed that $A$. baumannii cells exposed to $100 \mu \mathrm{g} / \mathrm{ml}$ PlyA suffered from extrusion (D) and rapid disruption at single (E) or multiple sites (F), resulting in the loss of cytoplasmic contents and ultimately the formation of ghost cell (G). Bar sizes: $200 \mathrm{~nm}$.

PlyA could kill logarithmic phase A. baumannii WHG3066 and $P$. aeruginosa WHG3012 cells rapidly in a dose-dependent manner (Figure 1B). Specifically, a reduction of over 5 logs (from $6.6 \log$ to $1.2 \log$ ) was observed after treating the A. baumannii cells with $50 \mu \mathrm{g} / \mathrm{ml}$ PlyA for $1 \mathrm{~h}$ (Figure 1B), and a reduction about $2.5 \operatorname{logs}$ (from $6.7 \log$ to $3.8 \mathrm{log}$ at $100 \mu \mathrm{g} / \mathrm{ml}$ PlyA) for $P$. aeruginosa cells (Figure 1B). Nearly no viable A. baumannii cells were detected when the concentration of PlyA increased to $100 \mu \mathrm{g} / \mathrm{ml}$. While its parental lysin OBPgp279 (with equimolar) could only cause a reduction of about 1.38 logs (data not shown). The time kill curve revealed that the viable A. baumannii cell numbers were reduced about 1.8 logs (from $6.6 \log$ to $4.8 \mathrm{log}$ ) within the first $15 \mathrm{~min}$ when treated with $50 \mu \mathrm{g} / \mathrm{ml} \mathrm{PlyA}$, and the killing continued for at least $60 \mathrm{~min}$ (Figure 1C).

Transmission electron microscope Images revealed that the A. baumannii WHG3066 cells exposed to PlyA suffered from leakage (Figure 1D) and rapid disruption at single (Figure 1E) or multiple sites (Figure 1F), resulting in the partial or total loss of cytoplasmic contents and ultimately loss of cell integrity (Figure 1G).

\section{Antibacterial Spectrum of PlyA}

Next, we tested the susceptibility of a collection of clinical isolates of A. baumannii and P. aeruginosa to PlyA (in Tris- $\mathrm{HCl}$ ), including another $31 \mathrm{~A}$. baumannii isolates and $35 \mathrm{P}$. aeruginosa isolates with multiple antimicrobial resistances profiles (Table 1). The plating assay revealed that PlyA was active against all A. baumannii isolates tested, causing a reduction of 1.0-4.2 logs. Except WHG3033, all $P$. aeruginosa isolates tested (35/36) were susceptible to PlyA (with a reduction of $0.5-2.7$ logs in viable cell number; Figure 2). The variable susceptibility of these clinical isolates to PlyA may be due to their different modifications in their outer membrane structure. No antibacterial activity of PlyA was observed against Escherichia coli BL21(DE3) and Bacillus cereus IS195 tested (data not shown). These results 


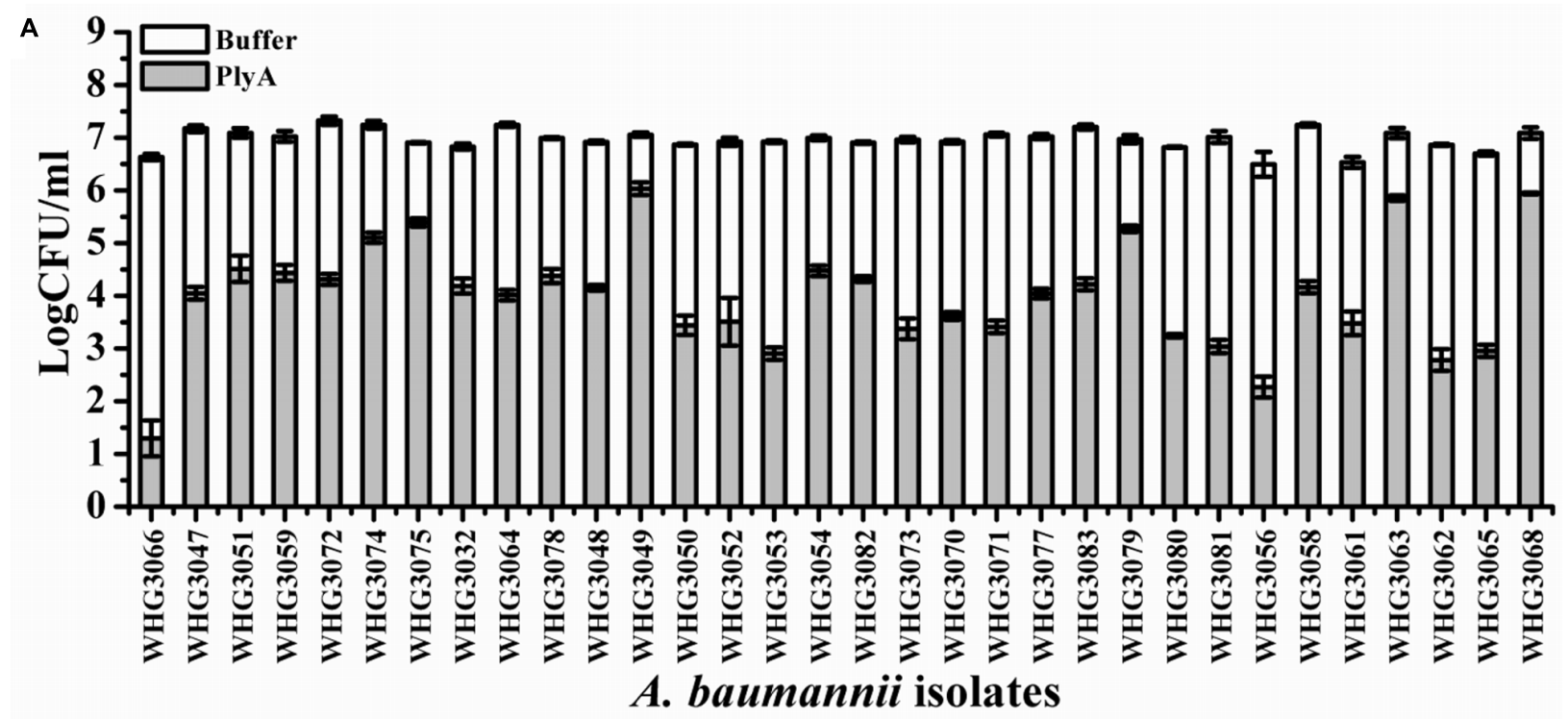

B

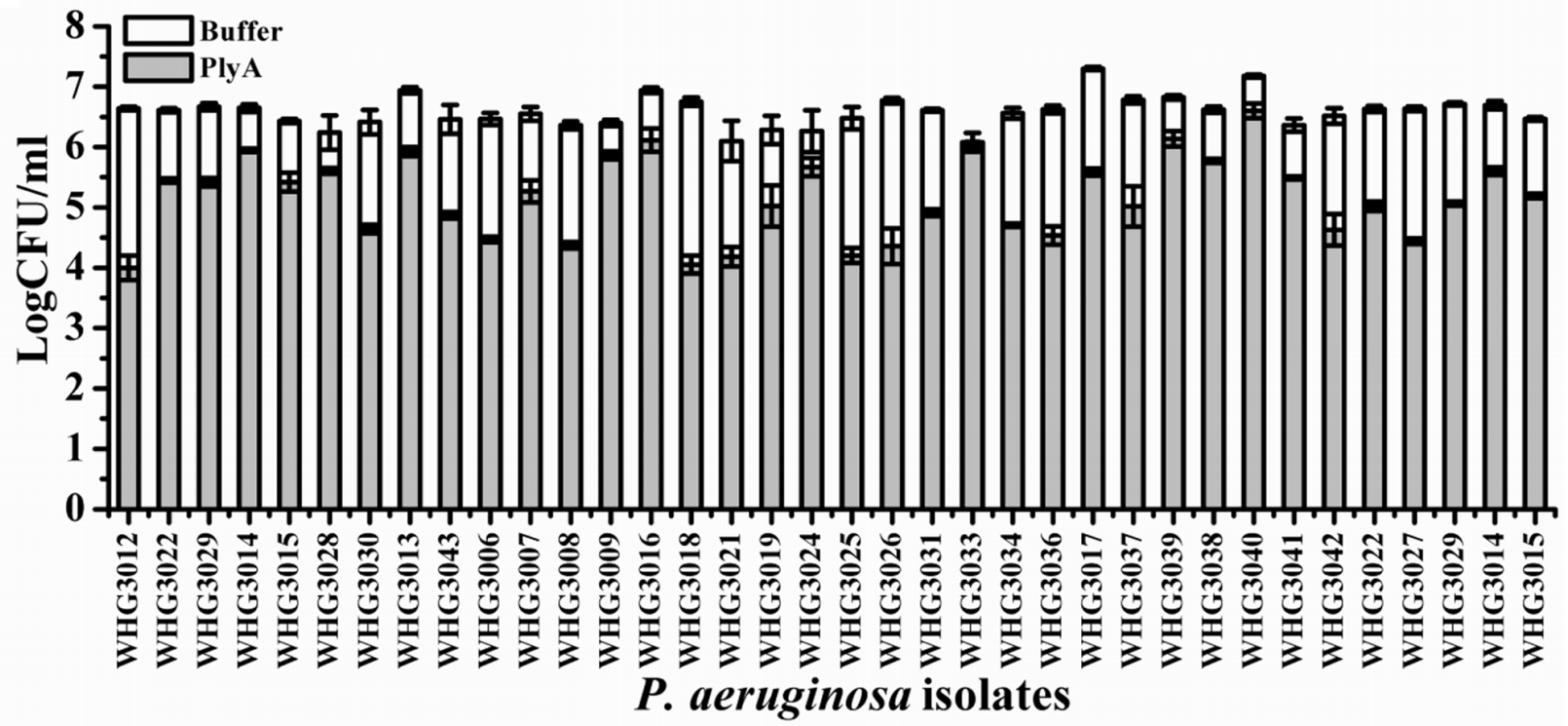

FIGURE 2 | Antibacterial activity of PlyA against clinical isolates of $\boldsymbol{A}$. baumannii and $\boldsymbol{P}$. aeruginosa in $20 \mathrm{mM}$ Tris-HCl (pH 7.4). (A) Antibacterial activity of PlyA (50 $\mu \mathrm{g} / \mathrm{ml})$ against $A$. baumannii clinical isolates in logarithmic phase at $37^{\circ} \mathrm{C}$ for $1 \mathrm{~h}$. (B) Antibacterial activity of PlyA (100 $\left.\mu \mathrm{g} / \mathrm{ml}\right)$ against $P$. aeruginosa clinical isolates in logarithmic phase at $37^{\circ} \mathrm{C}$ for $1 \mathrm{~h}$.

demonstrate that PlyA has broad antibacterial activity against MDR A. baumannii and $P$. aeruginosa isolates in Tris- $\mathrm{HCl}$ buffer.

\section{Bacterial Phase Affects the Antibacterial Activity of PlyA}

Although PlyA showed good antibacterial activity (from $6.8 \log$ to $1.8 \mathrm{log}$ ) against logarithmic phase A. baumannii (Figure 1C), only a minor activity (from $6.8 \mathrm{log}$ to $6.4 \mathrm{log}$ ) could be observed after treatment of the stationary phase A. baumannii WHG3066 cells with $50 \mu \mathrm{g} / \mathrm{ml}$ PlyA for $1 \mathrm{~h}$ (Figure 3A). Different susceptibilities to PlyA were also observed for logarithmic and stationary phase
P. aeruginosa WHG3012 cells, but the stationary cells were still killed with a reduction of approximately 2 logs in cell number (Figure 3B). These results show that stationary phase A. baumannii and $P$. aeruginosa cells are not or partially killed in comparison to their logarithmic phase ones, respectively.

\section{OMPs Enhance the Antibacterial Activity of PlyA Against Stationary Phase Cells}

Outer membrane permeabilizers (OMPs), such as EDTA and citric acid have been used to enhance the bacteriolytic activity of lysins (Oliveira et al., 2014). However, an unneglectable minor 

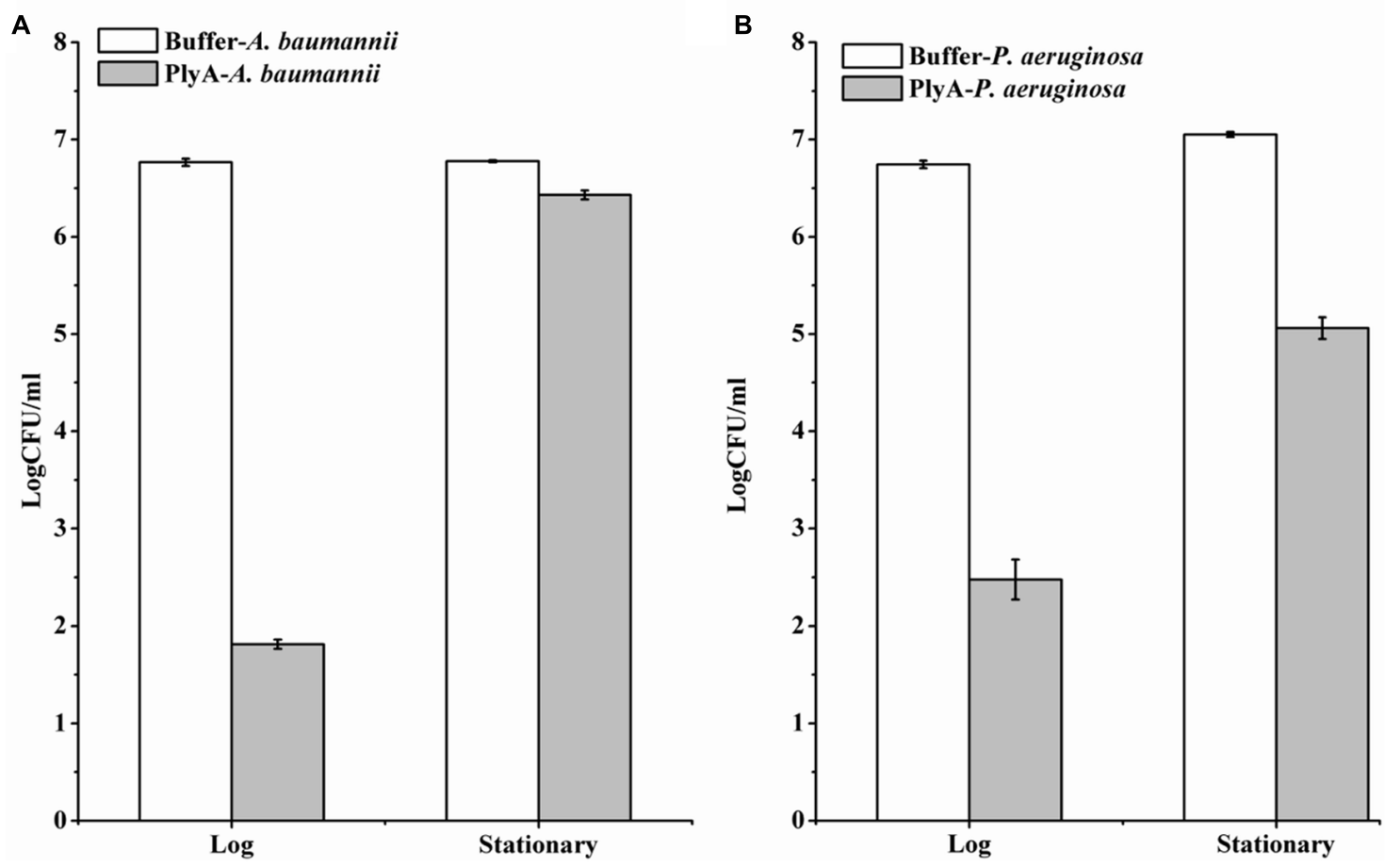

FIGURE 3 | Comparison between the activity of PlyA against $A$. baumannii WHG3066 (A) and $P$. aeruginosa WHG3012 (B) cells in logarithmic and stationary phase at $37^{\circ} \mathbf{C}$ for $1 \mathrm{~h}$ in $20 \mathrm{mM}$ Tris- $\mathrm{HCl}(\mathrm{pH}$ 7.4). Concentration of PlyA: $50 \mu \mathrm{g} / \mathrm{ml}$.

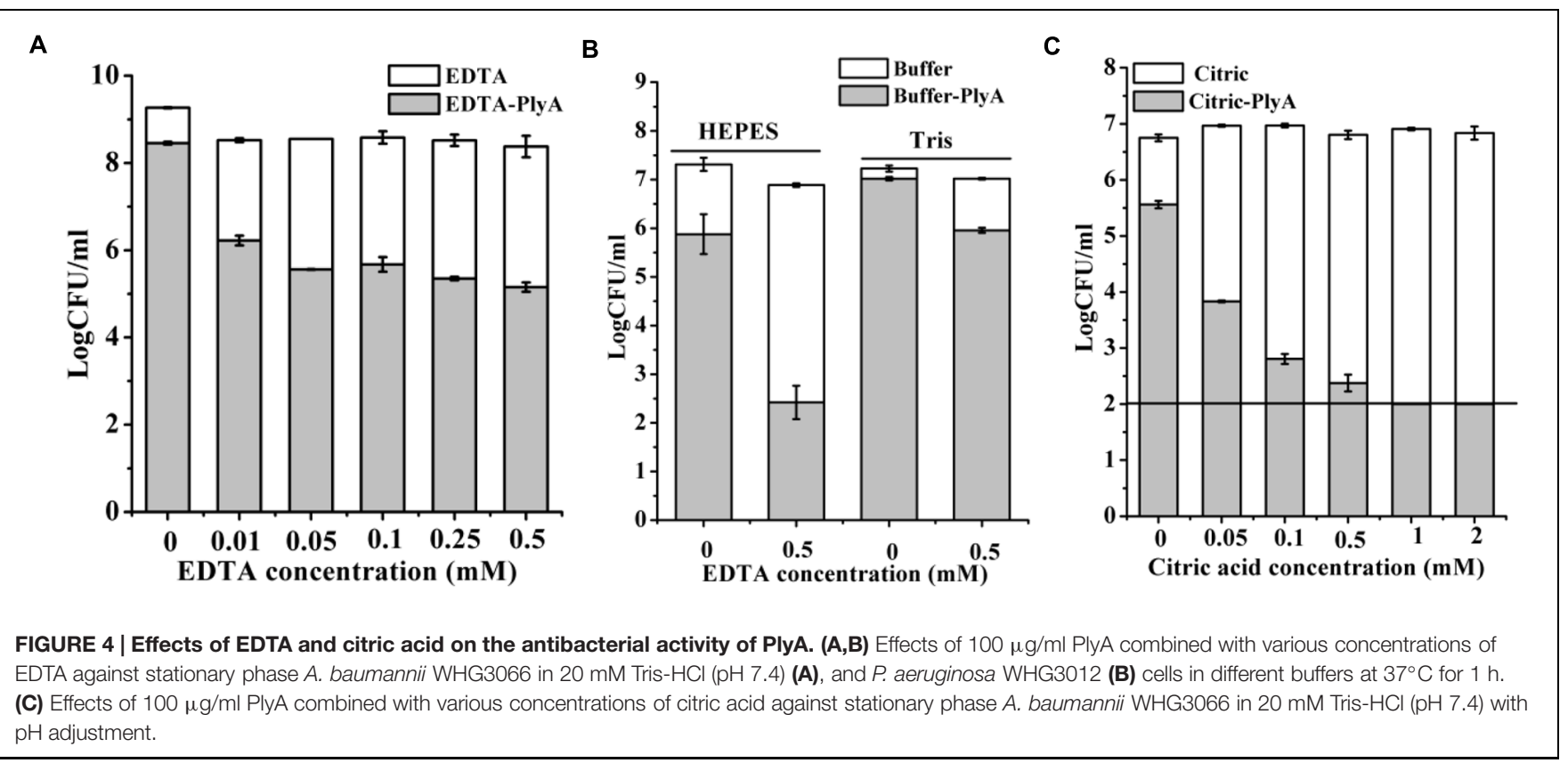

killing effect on logarithmic $P$. aeruginosa was noted in the presence of EDTA (Walmagh et al., 2012) and citric acid alone (Oliveira et al., 2014) previously by other researchers. Therefore, we only tested the synergism of these OMPs with PlyA against stationary phase cells. Figure $4 \mathrm{~A}$ showed that EDTA alone has a minor killing effect on stationary phase A. baumannii cells, with a reduction of only 0.3 logs in $20 \mathrm{mM}$ Tris- $\mathrm{HCl}$ ( $\mathrm{pH}$ 7.4). Whilst, an obvious enhanced antibacterial activity of PlyA was observed in the presence of EDTA, with a reduction of 2.3-3.2 logs in the viable cell number. The synergism between EDTA and PlyA was observed not only in $20 \mathrm{mM}$ Tris- $\mathrm{HCl}$ (pH 7.4), but also in $5 \mathrm{mM}$ HEPES-NaOH ( $\mathrm{pH}$ 7.4) against stationary 
phase $P$. aeruginosa (with a reduction of 4.4 logs; Figure 4B). Because the acidification effect of citric acid was reported to kill logarithmic cells (Oliveira et al., 2014; Briers and Lavigne, 2015), we tested the effect of citric acid (0.05-2 mM) on PlyA antibacterial activity against the stationary phase cultures of A. baumannii by adjusting the $\mathrm{pH}$ values of the mixtures to 7.4 after adding citric acid to the Tris-HCl buffer. Results showed that a citric acid dose-dependent synergism between citric acid and PlyA was observed (Figure 4C), indicating that mechanisms other than the acidification effect exist underlying the synergism between citric acid and PlyA against the stationary phase cells.

\section{Antibacterial Activity of PlyA in Bio-Matrix Conditions}

Finally, we evaluated the performance of PlyA in some biomatrix conditions, including LB culture medium, pasteurized milk, and human serum, which are more complicate than the pure Tris- $\mathrm{HCl}$ or HEPES-NaOH buffer. Figure 5 showed that no antibacterial activity was observed in the presence of PlyA in these conditions against logarithmic phase A. baumannii cells. The abolished activity was also observed in $\mathrm{MH}, \mathrm{BHI}$, and TSBN culture media (data not shown). These results indicate that PlyA alone is easily inactivated in complicate environments, limiting its application only to relatively simple or special conditions.

\section{DISCUSSION}

The global emergence of MDR bacteria is calling to find novel molecules for treating the infections caused by them. Some

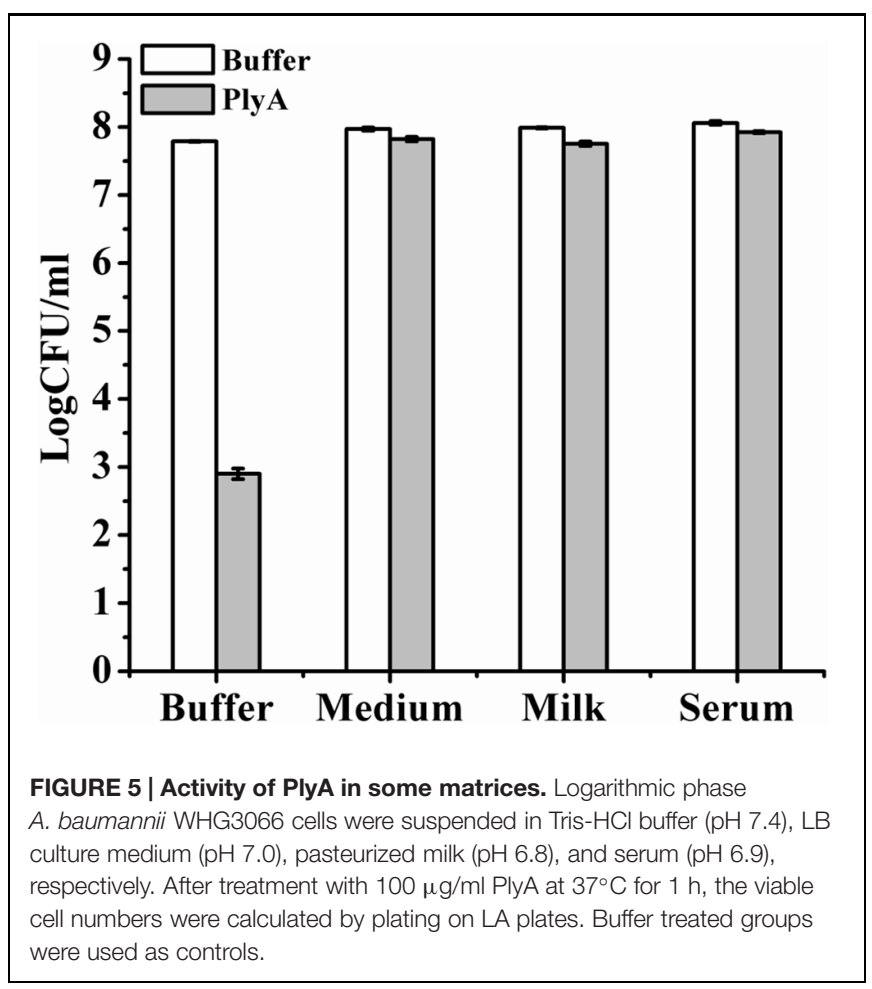

recent studies on peptide-modified lysins or prophage lysins with similar structural compositions have shown that they could kill Gram-negative pathogens, including P. aeruginosa (Briers et al., 2014a) and A. baumannii (Lood et al., 2015). However, less is known about the conditions that influence the activities of peptide-modified lysins and their potential limitations in real applications. In the present study, we found that the activity of a new peptide-modified lysin could be affected by the bacterial growth phase and the bio-matrix, which should be taken into consideration for the development of new peptide-modified lysins.

We observed that PlyA showed good antibacterial activity in Tris- $\mathrm{HCl}$ buffer against logarithmic phase A. baumannii and $P$. aeruginosa clinical isolates (Figure 2), including these with various antibiotic resistance profiles (Table 1 , some of them are MDR isolates). These results demonstrate that the strategy of modifying lysins with a selected peptide is powerful to obtain novel engineered lysins against Gram-negative bacteria, including MDR isolates. The action model of a well known peptide-modified lysin, Artilysin ${ }^{\circledR}$, is speculated to be: (1) the peptide fused in the $\mathrm{N}$ - or C-terminal of a target lysin interacts with the lipopolysaccharide of the Gram-negative bacteria, resulting in the destabilization and deformation of the outer membrane; (2) the lysin moiety transfers through the outer membrane driven by the self-promoted uptake of the peptide, and (3) thus gets access to and hydrolyze its peptidoglycan substrates, ultimately gives rise to cell lysis. This hypothesized mode of action was recently confirmed by Briers and coworkers in the time-lapse microscopy of $P$. aeruginosa cells exposed to Artilysin ${ }^{\circledR}$ LoGT-008 (Briers et al., 2014b). The TEM analysis of A. baumannii cells in this study also supports this hypothesis. As shown in Figure 1, PlyA disintegrates the cell wall of $A$. baumannii, and causes the loss of cytoplasmic contents in a single or multiple sites. This observation is quite similar with the typical phenomenon of osmotic-mediated cell lysis following the actions of phage lysins against Gram-positive bacteria reported elsewhere (Daniel et al., 2010).

Although PlyA showed good antibacterial activity against cells in logarithmic phase, a nearly abolished antibacterial activity was observed against stationary phase A. baumannii cells (Figure 3A). In case of stationary phase $P$. aeruginosa the antibacterial activity is not abolished but greatly reduced (Figure 3B). This observation is quite consistent with a recent report showing that a higher antibacterial activity of PlyF307 lysin was noted against exponentially growing A. baumannii (Lood et al., 2015). The different susceptibility may be due to the different structure and composition of the cell membranes between logarithmic and stationary phase cells. The outer membrane of bacteria is mainly composed of lipopolysaccharides, phospholipids and proteins, and their contents and types are varied in different environmental conditions and growth phases (Cronan, 1978). Additionally, there may be also difference in peptidoglycan architecture between logarithmic and stationary phase cells, such as the thickness of peptidoglycan layer, which influences their susceptibility to PlyA. 
The strong synergisms between PlyA and OMPs (EDTA and citric acid in Tris- $\mathrm{HCl}$ and HEPES-NaOH buffer) against stationary phase cultures of $A$. baumannii (Figures $4 \mathrm{~A}, \mathrm{C}$ ) and $P$. aeruginosa cells (Figure $4 \mathrm{~B}$ ) indicate that the outer membrane of Gram-negative bacteria is indeed a physical barrier for the bacteriolytic activity of natural lysins. By combining with OMPs, PlyA may be helpful in ex vivo and topical applications, such as environmental or surface disinfection, but not suitable for systemic infections due to the potential risk of anti-coagulating properties of the OMPs.

Other researchers have noted that protonated form of citric acid has both chelating effect and acidification effect on bacterial cells, and the acidification effect could damage the outer membrane of Gram-negative bacteria (such as P. aeruginosa) and cause a reduction in viable cell number (Oliveira et al., 2014; Briers and Lavigne, 2015). However, in the present study, we found that the acidification-killing effect of citric acid ( $2 \mathrm{mM})$ was only observed in logarithmic cultures of A. baumannii, but not stationary phase ones (data not shown). Moreover, an obvious synergism between citric acid and PlyA was observed against the stationary phase cultures of $A$. baumannii in a citric acid dosedependent manner when $\mathrm{pH}$ values were adjusted to neutral $\mathrm{pH}$ (Figure 4C). These results indicate that it is the chelating effect, but not acidification effect of citric acid, which enhanced the antibacterial activity of PlyA against stationary phase cultures of A. baumannii.

One serious shortcoming for PlyA may be its inactivation in complicate bio-matrices, including media, milk and serum (Figure 5), which means PlyA could only be used in relatively simple or specified conditions, such as material and skin surface disinfection. One possible reason for the inactivation of PlyA may be the conjugation and passivation of negatively charged molecules present in these matrices to the positively charged peptides, which may render the peptide losing the outer membrane penetrating activity.

However, in the previous reports, the efficacies of some Artilysin $^{\circledR} \mathrm{s}$ have been demonstrated in $P$. aeruginosa infected Caenorhabditis elegans model and human keratinocytes monolayer model in the presence of EDTA (Briers et al., 2014b), as well as in in vitro case studies of dog otitis caused by $P$. aeruginosa (Briers and Lavigne, 2015). Therefore,

\section{REFERENCES}

Bassetti, M., Villa, G., and Pecori, D. (2014). Antibiotic-resistant Pseudomonas aeruginosa: focus on care in patients receiving assisted ventilation. Future Microbiol. 9, 465-474. doi: 10.2217/fmb.14.7

Briers, Y., Cornelissen, A., Aertsen, A., Hertveldt, K., Michiels, C. W., Volckaert, G., et al. (2008). Analysis of outer membrane permeability of Pseudomonas aeruginosa and bactericidal activity of endolysins KZ144 and EL188 under high hydrostatic pressure. FEMS Microbiol. Lett. 280, 113-119. doi: 10.1111/j.1574-6968.2007. 01051.x

Briers, Y., and Lavigne, R. (2015). Breaking barriers: expansion of the use of endolysins as novel antibacterials against Gram-negative bacteria. Future Microbiol. 10, 377-390. doi: 10.2217/fmb.15.8

Briers, Y., Volckaert, G., Cornelissen, A., Lagaert, S., Michiels, C. W., Hertveldt, K., et al. (2007). Muralytic activity and modular structure of the endolysins of it seems that it is not easy and straight-forward to design an engineered peptide-modified lysin which shows robust activity under infection conditions as described for $\operatorname{Artilysin}^{\circledR}$ s. Some optimization like fusing different peptides with different endolysins and combining with different linkers is required to obtain an antibacterial lysin with the desired properties and a robust activity (Briers et al., 2014b).

\section{CONCLUSION}

We report here a newly engineered lysin, PlyA, with high bacteriolytic activity against $A$. baumannii and $P$. aeruginosa in vitro. This study also indicated that conditions such as bacterial growth phase and the bio-matrix can influence the antibacterial activity of PlyA, suggesting that there are still some limitations that should be taken into consideration for the development of new peptide-modified lysins, and optimization is needed to obtain an antibacterial lysin with robust antibacterial activity.

\section{AUTHOR CONTRIBUTIONS}

HY and HW conceived the study. HY and MW performed experiments. HY, JY, and HW analyzed data. HY and HW wrote the paper.

\section{ACKNOWLEDGMENTS}

This work was supported by the National Natural Science Foundation of China (No. 31400126, 31570175), the Basic Research Program of the Ministry of Science and Technology of China (No. 2012CB721102), the Chinese Academy of Sciences (No. KJZD-EW-L02), the Open Research Fund Program of the State Key Laboratory of Virology of China (No. 2014IOV002), and the Key Laboratory of Emerging Infectious Diseases and Biosafety in Wuhan.

We are grateful to Ms. Pei Zhang for her excellent technical support in microscope analysis.

Pseudomonas aeruginosa bacteriophages phiKZ and EL. Mol. Microbiol. 65, 1334-1344. doi: 10.1111/j.1365-2958.2007.05870.x

Briers, Y., Walmagh, M., Grymonprez, B., Biebl, M., Pirnay, J. P., Defraine, V., et al. (2014a). Art-175 is a highly efficient antibacterial against multidrugresistant strains and persisters of Pseudomonas aeruginosa. Antimicrob. Agents Chemother. 58, 3774-3784. doi: 10.1128/AAC.02668-14

Briers, Y., Walmagh, M., Van Puyenbroeck, V., Cornelissen, A., Cenens, W., Aertsen, A., et al. (2014b). Engineered endolysin-based "Artilysins" to combat multidrug-resistant gram-negative pathogens. MBio 5:e1379-14. doi: 10.1128/mBio.01379-14

Briers, Y., Walmagh, M., and Lavigne, R. (2011). Use of bacteriophage endolysin EL188 and outer membrane permeabilizers against Pseudomonas aeruginosa. J. Appl. Microbiol. 110, 778-785. doi: 10.1111/j.1365-2672.2010. 04931.x

Cronan, J. E. Jr. (1978). Molecular biology of bacterial membrane lipids. Annu. Rev. Biochem. 47, 163-189. doi: 10.1146/annurev.bi.47.070178.001115 
Daniel, A., Euler, C., Collin, M., Chahales, P., Gorelick, K. J., and Fischetti, V. A. (2010). Synergism between a novel chimeric lysin and oxacillin protects against infection by methicillin-resistant Staphylococcus aureus. Antimicrob. Agents Chemother. 54, 1603-1612. doi: 10.1128/AAC.01625-09

Fischbach, M. A., and Walsh, C. T. (2009). Antibiotics for emerging pathogens. Science 325, 1089-1093. doi: 10.1126/science.1176667

Fischetti, V. A. (2008). Bacteriophage lysins as effective antibacterials. Curr. Opin. Microbiol. 11, 393-400. doi: 10.1016/j.mib.2008.09.012

Garcia-Quintanilla, M., Pulido, M. R., Lopez-Rojas, R., Pachon, J., and Mcconnell, M. J. (2013). Emerging therapies for multidrug resistant Acinetobacter baumannii. Trends Microbiol. 21, 157-163. doi: 10.1016/j.tim.2012.12.002

Huang, Y., Yang, H., Yu, J., and Wei, H. (2015). Molecular dissection of phage lysin PlySs2: integrity of the catalytic and cell wall binding domains is essential for its broad lytic activity. Virol. Sin. 30, 45-51. doi: 10.1007/s12250-0143535-6

Knoll, B. M., and Mylonakis, E. (2014). Antibacterial bioagents based on principles of bacteriophage biology: an overview. Clin. Infect. Dis. 58, 528-534. doi: $10.1093 / \mathrm{cid} / \mathrm{cit} 771$

Lai, M. J., Lin, N. T., Hu, A., Soo, P. C., Chen, L. K., Chen, L. H., et al. (2011). Antibacterial activity of Acinetobacter baumannii phage varphiAB2 endolysin (LysAB2) against both gram-positive and gram-negative bacteria. Appl. Microbiol. Biotechnol. 90, 529-539. doi: 10.1007/s00253-011-3104-y

Li, X. Z., Plesiat, P., and Nikaido, H. (2015). The challenge of efflux-mediated antibiotic resistance in Gram-negative bacteria. Clin. Microbiol. Rev. 28, 337-418. doi: 10.1128/CMR.00117-14

Lim, J. A., Shin, H., Heu, S., and Ryu, S. (2014). Exogenous lytic activity of SPN9CC endolysin against gram-negative bacteria. J. Microbiol. Biotechnol. 24, 803-811.

Livermore, D. M., and Woodford, N. (2006). The beta-lactamase threat in Enterobacteriaceae, Pseudomonas and Acinetobacter. Trends Microbiol. 14, 413-420. doi: 10.1016/j.tim.2006.07.008

Lood, R., Winer, B. Y., Pelzek, A. J., Diez-Martinez, R., Thandar, M., Euler, C. W., et al. (2015). Novel phage lysin capable of killing the multidrug-resistant gramnegative bacterium Acinetobacter baumannii in a mouse bacteremia model. Antimicrob. Agents Chemother. 59, 1983-1991. doi: 10.1128/AAC.04641-14

Lukacik, P., Barnard, T. J., Keller, P. W., Chaturvedi, K. S., Seddiki, N., Fairman, J. W., et al. (2012). Structural engineering of a phage lysin that targets gram-negative pathogens. Proc. Natl. Acad. Sci. U.S.A. 109, 9857-9862. doi: 10.1073/pnas.1203472109

Morita, M., Tanji, Y., Orito, Y., Mizoguchi, K., Soejima, A., and Unno, H. (2001). Functional analysis of antibacterial activity of Bacillus amyloliquefaciens phage endolysin against Gram-negative bacteria. FEBS Lett. 500, 56-59. doi: 10.1016/S0014-5793(01)02587-X

Nelson, D. C., Schmelcher, M., Rodriguez-Rubio, L., Klumpp, J., Pritchard, D. G., Dong, S., et al. (2012). Endolysins as antimicrobials. Adv. Virus Res. 83, 299-365. doi: 10.1016/B978-0-12-394438-2.00007-4

Oliveira, H., Thiagarajan, V., Walmagh, M., Sillankorva, S., Lavigne, R., Neves-Petersen, M. T., et al. (2014). A thermostable Salmonella phage endolysin, Lys68, with broad bactericidal properties against gramnegative pathogens in presence of weak acids. PLOS ONE 9:e108376. doi: 10.1371/journal.pone.0108376

Pastagia, M., Schuch, R., Fischetti, V. A., and Huang, D. B. (2013). Lysins: the arrival of pathogen-directed anti-infectives. J. Med. Microbiol. 62, 1506-1516. doi: 10.1099/jmm.0.061028-0

Potron, A., Poirel, L., and Nordmann, P. (2015). Emerging broad-spectrum resistance in Pseudomonas aeruginosa and Acinetobacter baumannii: mechanisms and epidemiology. Int. J. Antimicrob. Agents 45, 568-585. doi: 10.1016/j.ijantimicag.2015.03.001

Silvestro, L., and Axelsen, P. H. (2000). Membrane-induced folding of cecropin A. Biophys. J. 79, 1465-1477. doi: 10.1016/S0006-3495(00)76398-3

Walmagh, M., Briers, Y., Dos Santos, S. B., Azeredo, J., and Lavigne, R. (2012). Characterization of modular bacteriophage endolysins from Myoviridae phages OBP, 201phi2-1 and PVP-SE1. PLoS ONE 7:e36991. doi: 10.1371/journal.pone.0036991

Yang, H., Wang, D. B., Dong, Q. H., Zhang, Z. P., Cui, Z. Q., Deng, J. Y., et al. (2012). Existence of Separate Domains in Lysin PlyG for Recognizing Bacillus anthracis Spores and Vegetative Cells. Antimicrob. Agents Chemother. 56, 5031-5039. doi: 10.1128/AAC.00891-12

Yang, H., Yu, J., and Wei, H. (2014). Engineered bacteriophage lysins as novel anti-infectives. Front. Microbiol. 5:542. doi: 10.3389/fmicb.2014.00542

Conflict of Interest Statement: The authors declare that the research was conducted in the absence of any commercial or financial relationships that could be construed as a potential conflict of interest.

Copyright (c) 2015 Yang, Wang, Yu and Wei. This is an open-access article distributed under the terms of the Creative Commons Attribution License (CC BY). The use, distribution or reproduction in other forums is permitted, provided the original author(s) or licensor are credited and that the original publication in this journal is cited, in accordance with accepted academic practice. No use, distribution or reproduction is permitted which does not comply with these terms. 